\title{
GALAXIES, CLUSTERS AND FLUCTUATIONS
}

\author{
Richard Schaeffer \\ Service de Physique Théorique, \\ Institut de Recherche Fondamentale, \\ CEA-CEN-Saclay, 91191 Gif-sur-Yvette Cedex, France
}

\section{Abstract}

The luminosity function of galaxies and clusters as well as their correlations can be calculated assuming the many-body correlation functions are scale invariant. The same hypothesis leads to predictions on the fractal dimension of the galaxy distribution. The latter is found to be bifractal that is characterized by two dimensions $D=3-\gamma$ in the cluster region, and $D=(3-\gamma)(2+\alpha)$ in the nearly empty regions, $\alpha$ being the index introduced by Schechter for the galaxy luminosity function. Finally, the same models lead to predictions for the evolution of the cluster and $x$-ray luminosity functions, as well as for the Sunyaev-Zeldovich effect due to all virialized clusters, which is found to be large and to produce fluctuations of order $10^{-5}$ at sub-arc minute scalar in the microwave background.

\section{GALAXY AND MATTER CORRELATIONS}

It is well-known that galaxies are strongly correlated at scales below 8 or $10 \mathrm{~h}^{-1} \mathrm{Mpc}$. Their observed correlation function can be fitted by a power law (Davis and Peebles 1983) : $\xi(r) \sim r^{-\gamma}$. At least in an $\Omega=1$ universe, such a scale invariant correlation function is expected (Peebles 1980) from theory, and the same argument can give its time (redshift) dependence (Peebles 1980) : $\xi \propto(1+2)^{\gamma-3}$, and predict that the $\mathrm{N}$-body correlation function scales as

$$
\xi_{N}\left(r_{1}, \ldots, r_{N}\right)=\lambda^{\gamma(N-1)} \xi_{N}\left(\lambda r_{1}, \ldots, \lambda r_{n}\right)
$$

Within this model, scaling laws can be established. The probability to find no galaxy within a given volume $V, P_{0}(V)$ has the form (White 1979, Sharp 1981, Fry 1984, Schaeffer 1984, 1987, Balian and Schaeffer 1987)

$$
\mathrm{P}_{0}(\mathrm{~V})=\exp -\mathrm{nV} \sigma(\mathrm{q})
$$

where $\mathrm{n}$ is the number density of galaxies, and

$$
q=n V \int \frac{V d^{3} r_{1}}{V} \int \frac{V d^{3} r_{2}}{V} \xi\left(r_{12}\right)=n V \bar{\xi}
$$

J. Audouze et al. (eds.), Large Scale Structures of the Universe, 215-220.

(c) 1988 by the IAU. 
is the scaling variable. The function $\sigma(q)$ is arbitrary.

Once a specific hierarchical model is chosen, assuming it holds for the matter distribution, the probability of having $\mathrm{N}$ objects in a volume $V$, and whence a given total mass within $V$, can be calculated leading to a prediction for the galaxy luminosity function. For the model (6), the galaxy luminosity function is approximately (Schaeffer 1987)

$$
\rho(L) d L \propto L_{0} / L \frac{1}{(\nu+1) !}\left[\ln \left(1+L_{0} / L\right)\right]^{v+1} e^{-L / L_{0}} \rho_{L} d L / L_{0}^{2}
$$

with $L_{0}=a q$, where a is a numerical factor and $q$ is to be calculated for a volume corresponding to galaxies. A reasonable choice is a sphere of $\sim 0.1 \mathrm{Mpc}$ radius, that is seen to produce a luminosity function quite close to the observations as compiled by Bahcall (1979). More recently, it has

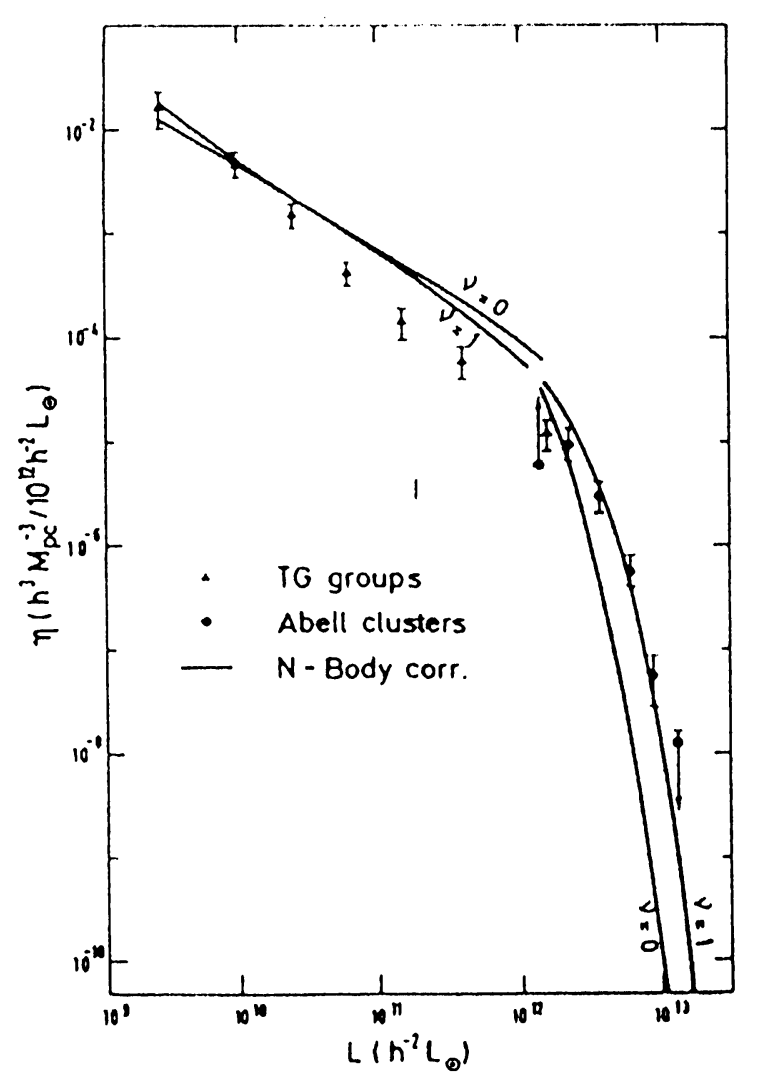

Fig. 1: Cluster luminosity function (Schaeffer 1987). The full lines are the predictions of the hierarchical clustering model for the non-linear matter distribution. The data result from a compilation by Bahcall (1979) of the luminosity distribution of Turner-Gott groups and Abel clusters. 
been shown (Balian and Schaeffer 1987) that

$$
\sigma(q) \sim q^{-\omega} \quad(0 \leqslant \omega<1)
$$

for large $q$ implies

$$
\varphi(\mathrm{L}) \mathrm{dL} \sim\left(\mathrm{L} / \mathrm{L}_{0}\right)^{\omega-2} \mathrm{dL}
$$

at small L. These two limits are similar simply because the limit of small $\mathrm{L} / \mathrm{L}_{0}$ is formally equivalent to the limit of large $\mathrm{q}$ in (11). They provide a relation between the large scale behaviour of the probability of holes and the divergence of the galaxy luminosity function at the faint end

$$
\omega=2+\alpha
$$

Note that current values of $\alpha \approx-1.2$ imply $\omega \sim 0.8$. But the model of Schaeffer (1984) leads to $\sigma(q) \sim q^{-1}(\ell n q)^{v+2}$, that is $\omega \approx 1$, and reproduces the data nearly as well as the Schechter parametrization.

In a similar way, the cluster distribution can be calculated, by assuming that either the galaxy or the matter distribution is given by (4). Both assumptions lead to the same result in the limit where clusters are much larger than galaxies. The predictions (Schaeffer 1987) using the model (6) are given by eq. (9), but for a value of $q$ adapted to clusters (e.q. calculated within a sphere of radius $1.5 \mathrm{~h}^{-1} \mathrm{Mpc}$ for Abell clusters, or imposing some density contrast for Turner-Gott groups). It can be seen that the same value $v \sim 1$ reproduces the data (Fig. 1).

\section{FRACTAL DIMENSION OF THE GALAXY DISTRIBUTION}

We present here the predictions that can be made (Balian and Schaeffer 1987) under the sole assumption, eq.(1), that the galaxy correlation functions are scale invariant, with a unique power law index $r$. As in any fractal system, there is a lbwer scale as well as an upper scale at which the scale invariance breaks down. For the galaxy distribution, the lower scale can be set as being the average distance $\ell_{c}$ between galaxies within a cluster. The largest possible scale where scale invariance is to be expected is $\ell_{0}$, the diameter of a sphere whose radius is the correlation length $r_{0} \sim 5 h^{-1} \mathrm{Mpc}$ that is $\ell_{0} \sim 10 \mathrm{~h}^{-1} \mathrm{Mpc}$. For $\mathrm{n} \sim 210^{-2} \mathrm{~h}^{3} \mathrm{Mpc}^{-3}$, that is counting only the bright galaxies, we have $\ell_{c} \sim 0.5 \mathrm{~h}^{-1} \mathrm{Mpc}$. For larger values of $\mathrm{n}, \ell_{\mathrm{c}}$ may be much smaller. The question is then whether or not in the limits

$$
\ell_{c}<\ell<\ell_{0}
$$

the galaxy distribution is, under the assumption (1), a fractal characterized by a unique fractal dimension $D=3-\gamma$. The answer is rather surprising, as can already be seen by calculating the Hausdorff dimension that is obtained by considering boxes of size $\ell$ and counting all occupied boxes. The number of the latter scales as $\ell^{-D_{0}}$, where $D_{0}$ is the Hausdorff dimension. Despite the scale invariance of the correlation function, we find

$$
D_{0}=(3-\gamma) \omega
$$

where $\omega$ has been considered in the previous section and reflects the scaling of the probability of holes at large scales. So, unless $\omega=1, D_{0}$ is different from the naive conclusion drawn from the observation of the power-law behaviour of the correlation function. The result (20) is valid 
at scales much larger than the distance $\ell_{c}$ of galaxies in a cluster, and much smaller than $\ell_{\mathrm{v}}$, the typical void size, defined as the scale at which the probability of holes starts to get sizeably below 1 .

This result can be understood provided we get some deeper insight into the problem. Instead of counting all occupied boxes, let us separate them into subclasses. We distinguish the boxes by the scaling of the number of galaxies they contain. It happens that it scales as a power-law

$$
\mathrm{N}(\ell) \sim \mathrm{A} \ell^{-\alpha}
$$

but, depending on the region of space we consider, $\alpha$ may take different values. Defining the number of baxes $\mathcal{N}_{\alpha}$ that scale as $\ell^{-\alpha}$, we find that the latter, as for the Hausdorff dimension, scales as a power-low of the box size

$$
N_{\alpha} \sim \ell^{-P(\alpha)},
$$

but the power is different for different values of $\alpha$. The model (3) for the correlation functions shows there are two very distinct populations of galaxies. Firstly those for which the number of galaxies within an occupied cell is a constant independent of the box size $(\alpha=0)$. This corresponds to isolated galaxies or groups. And secondly those for which the number of galaxies scales as $\ell^{3-\gamma}$ with the box size $(\alpha=3-\gamma)$, corresponding to sampling boxes that are situated within a cluster. The dimension of these two regions can be found by counting the number of boxes of each kind, and its scaling, eq. 23, with the box size. We find that the volume occupied by isolated galaxies scales with a dimension

$$
\begin{gathered}
f_{1 s 0} \sim(3-\gamma) \omega \\
\alpha_{1 \text { so }} \sim 0
\end{gathered}
$$

whereas the volume occupied by clusters scales as

$$
\begin{aligned}
& f_{c 1} \sim 3-\gamma \\
& \alpha_{c 1} \sim 3-\gamma
\end{aligned}
$$

This analysis was motivated by the conclusions drawn by Jones et al. (1987) from the CfA sample. The conclusions obtained here differ considerably from theirs. For a galaxy distribution that leads to a sufficiently large probability of holes $(\omega \sim 1)$ at large scales in order that the scaling region $\ell_{\mathrm{c}} \ll \ell<\ell_{\mathrm{v}}$ exists, it can be shown that the standard procedure for calculating $\alpha$ and $f(\alpha)$ does not lead to values that correspond to the definitions (10) and (11). This is discussed at length in Balian and Schaeffer (1987). If within our scaling model we nevertheless use the standard procedure (that is incorrect in our case) we get a whole spectrum of values of $\alpha$ and $f(\alpha)$ that are in a range compatible with the results of Jones et al (1987) instead of the two values (12) and (13) obtained with the new procedure. The analysis of the CfA sample with this new procedure (that is always valid and garanties that the scaling laws (10) and (11) are verified by the values of $\alpha$ and $f$ that are obtained) remains to be done.

\section{CLUSTER EVOLUTION}

It is possible from a model of the high order correlation function to construct a cluster luminosity function, eq. (4), that reproduces the observa- 
tions. The prediction of the theory is not only the shape of the luminosity function, but also the value of $\mathrm{L}_{0}$ is related to the luminosity density $\rho_{\mathrm{L}}$, the volume $\mathrm{V}$ of the cluster and the average correlation function within that volume. It happens that the evolution with redshift of all these quantities is known. It is thus possible to infer (Schaeffer and Silk 1987) the evolution of the cluster luminosity function with redshift, at least for the model $(6)$.

The luminosity function virialized clusters is readily deduced from eq. (4). Virialized clusters must have a radius $R$ such as

$$
\mathrm{R}<\left(\mathrm{L} / \mathrm{L}_{\mathrm{v}}\right)^{1 / 3} \frac{1}{1+\mathrm{z}} \mathrm{h}^{-1} \mathrm{Mpc}
$$

with $\mathrm{L}_{\mathrm{v}} \sim 2.510^{11} \mathrm{~h}^{-2} \mathrm{~L}_{\odot}$. We then get from eq. (8) the luminosity function for virialized clusters

$$
\begin{gathered}
\varphi(L) d L=\varphi_{0}[\ln (1+x)]^{\nu+1} / x d L \\
x=0.4(1+z)^{3-\gamma}\left(\frac{L}{L_{\nu}}\right)^{\gamma / 3}
\end{gathered}
$$

It shows a considerable spread in luminosities, with a large number of small objects of low luminosity. At large luminosities there is an exponential cut at

$$
\text { L. } ~ 1.210^{12}(1+\mathrm{z})^{-3(3-\gamma) / \gamma} \mathrm{h}^{-3} \mathrm{~L}_{\odot}
$$

Despite it is weaker than predicted by linear theory (Kaiser 1986), this evolution is fairly strong since $L_{*} \propto(1+z)^{-2}$. Despite the large spreading of $L$, it might be measurable. Expressions $(15-16)$ are valid up to a redshift $\mathrm{Z}_{\max }$ at which the scales (14) turn non-linear.

The Sunyaev-Zeldovich (1981) effect for one cluster depends on the cluster temperature, luminosity and radius $\Delta \mathrm{T} / \mathrm{T} \sim \mathrm{TLR}^{-2}$ assuming that the gaz density scales as the ratio of optical luminosity to cluster volume. The form (4) can then be used to compute the average $y$ of $\Delta T / T$ along a lign of sight and its fluctuations. From the redshift dependence of $L_{0} i t$ can be seen that numerous clusters are expected even at a fairly high redshift. A straighforward calculation then shows that there are usually several clusters contributing to $\mathrm{y}$ which is predicted to be fairly large

$$
y \sim 0.7\left(\frac{\Delta T}{T}\right)_{\text {coma }}\left(1+z_{\max }\right)^{3(3 \gamma-4) / 4}
$$

The typical size of the clusters that contribute to the counts is fairly small, much smaller than the size of Abell clusters or Turner-Gott groups. For $z_{\max } \sim 5, \mathrm{R}$ is of the order of $0.1 \mathrm{Mpc}$. The typical angular scale associated to these clusters is a fraction of an arc-minute. This is why the fluctuation below this angular scale are rather large, of the order of a few times the $\Delta T / T$ of Coma. The X-ray luminosity can be computed in a similar way, to get the cluster contribution from their mass function extrapolated at non-zero $z$ for times where clustering is still strong and cannot be calculated within linear theory. 
IV. CONCLUSION

The description of the galaxy and cluster distribution as they are at the present epoch requires statistics where the correlation functions are strong to all orders. The simple assumption that the many-body correlation functions are scale invariant allows to reproduce many observed properties of galaxies. Their luminosity function and its power-law behaviour at the faint end can be obtained. It is intimately (and surprisingly : this is one of the musteries of scale invariance) related to the presence of holes at large scales. This power-law behaviour is also related to the Hausdorff dimension of the galaxy distribution. Similarly the cluster luminosity function can be constructed, and especially the exponential fall-off at large luminosities is obtained. The scale-invariant model for the galaxy correlation functions thus represents a good description of galaxy clustering. It is only a model, since the derivation of the many-body galaxy correlation function from first principle is, despite valuable efforts, still a problem. This description can nevertheless be used to predict the evolution of clusters, to calculate the $x$-ray background and the contribution of the Sunyaev-Zeldovich effect to the microwave background fluctuations, with a degree of confidence that could never be achieved before.

The fractal dimensions of the galaxy distribution were calculated with $\mathrm{R}$. Balian. Also, the prediction for $x$-ray background and the microwave fluctuations were obtained in collaboration with J. Silk. I would like to thank them for many discussions.

\section{$\underline{\text { References }}$}

BALIAN R., SCHAEFFER R. (1987) to be published

BAHCALL N.A. (1979) Ap. J. 232, 689

DAVIS M., PEEBLES P.J.E. (1983) Ap. J. 267, 465

FRY J. (1984) Ap. J. Lett. 277, L5

JONES B.J.T., MARTINEZ V.J., SAAR E., EINASTO J. (1987) to be published KAISER N. (1986) MNRAS 222, 323

PEEBLES P.J.E. (1980), The large Scale Structure of the Universe, Princeton University Press, Princeton, N.J.

SCHAEFFER R. (1984) A.A. Lett. 134, L15

SCHAEFFER R. (1987) A.A. Lett. 180, L5

SCHAEFFER R., SILK J. (1987) to be published

SHARP N. (1981) M.N.R.A.S. 195, 857

SUNYAEV R.A., ZELDOVICH Ya. B. (1981) Ap. Sp. Phys. Rev. 1, 1

WHITE S.DM. (1979) M.N.R.A.S. 186, 145 